\section{OPEN SCIENCE POR DEFECTO. LA NUEVA NORMALIDAD PARA LA INVESTIGACIÓN}

\author{
Eva Méndez \\ Universidad Carlos III de Madrid. \\ ORCID: https://orcid.org/0000-0002-5337-4722 \\ emendez@bib.uc3m.es
} OPEN SCIENCE BY DEFAULT. THE
"NEW NORMAL" FOR RESEARCH

Cómo citar este artículo/Citation: Méndez, Eva (2021). Open Science por defecto. La nueva normalidad para la investigación. Arbor, 197(799): a587. https://doi.org/10.3989/arbor.2021.799002

Recibido: 14 septiembre 2020. Aceptado: 22 diciembre 2020. Publicado: 9 abril 2021

RESUMEN: Este trabajo aborda el nuevo paradigma de la Open Science o ciencia en abierto desde la perspectiva europea, pero destacando su necesario alcance global. Se analiza el concepto, origen y evolución de la Open Science y se discuten sus retos y la demora de su completa implementación. Se parte de la hipótesis de que la Open Science debería de ser el paradigma de comunicación científico por defecto en el siglo XXI. En primer lugar, se revisa el concepto y alcance de Open Science frente a Open Access y en su interacción con Open Knowledge, así como la necesidad de mantener la denominación como «marca». En segundo lugar, se recogen los requisitos, reflexiones y parámetros para hacer de la ciencia un bien común y se describen los retos de la Open Science en Europa, que se han convertido en los principales pilares y/o elementos constitutivos de la ciencia en abierto. Se reflexiona sobre su puesta en práctica, detallando dos aproximaciones: 1) la creación de políticas y estrategias formales a nivel Estados miembros (top-down), y 2) la implementación bottom-up a través de Compromisos Prácticos de Implementación (PCls). Asimismo, se destacan los problemas reales que alejan a los agentes implicados de protagonizar el cambio sistémico hacia la Open Science. Finalmente se reflexiona sobre el efecto de la COVID-19 que ha corroborado la necesidad urgente de la ciencia en abierto. Este artículo recoge, a modo de ensayo, la experiencia y el trabajo realizado en los últimos cuatro años en la European Open Science Policy Platform, donde la autora ha presidido el segundo mandato desde 2018 a 2020. La autora expone algunas de sus principales teorías en torno a la Open Science y defiende su tesis de los compromisos prácticos de implementación y la confluencia de aproximaciones de prácticas de los agentes implicados y políticas de ciencia abierta, así como su categórica aproximación a la Open Science como marca, actitud y derecho.

PALABRAS CLAVE: Open Science, ciencia en abierto, política científica, acceso abierto, conocimiento abierto, COVID-19, Europa.
Copyright: (C) 2021 CSIC. Este es un artículo de acceso abierto distribuido bajo los términos de la licencia de uso y distribución Creative Commons Reconocimiento 4.0 Internacional (CC BY 4.0).

ABSTRACT: This work addresses the new paradigm of Open Science (OS) in Europe but taking into account its global reach. It analyses the origin, scope, and evolution of Open Science along with the policies, recommendations, and conditions for the dissemination of scientific knowledge, discussing the delay in OS's full implementation. First, the concept and scope of Open Science versus Open Access and its interaction with Open Knowledge/Scholarship is reviewed, as well as the need to maintain the name as a "brand». Second, we describe the requirements, reflections, and parameters to make science a common good. Also reviewed are the challenges of Open Science in Europe, which have become the main pillars and/or constituent elements of Open Science. The implementation of Open Science is discussed detailing two approaches: 1 ) the creation of formal policies and strategies at Member State level (top-down), and 2) the bottom-up implementation by stakeholders through Practical Commitments for Implementation (PCls). Likewise, the real problems that prevent stakeholders from leading systemic change towards Open Science are highlighted. Finally, the paper reflects on the effect of COVID-19, which has underlined the urgent need for Open Science. This article gathers, as an essay, the experience and work carried out over the last four years in the European Open Science Policy Platform, where the author has been chair in the second mandate of the platform (2018-2020), as well as some of the main theories and developments of Open Science, while reflecting the state of the art at the end of the Horizon2020 European framework program.

KEYWORDS: Open Science, Scientific Policy, Open Access, Open Knowledge, COVID-19, Europe. 


\section{INTRODUCCIÓN Y CONTEXTO}

La investigación es cada vez más compleja, más digital y más basada en los datos y en las grandes capacidades de computación, lo que ha dado lugar a la eResearch (eCiencia en español) y a la computación científica. Asimismo, las tecnologías digitales, particularmente la World-Wide Web que facilita una conducta distribuida de investigación colaborativa (David, Den Besten y Schroeder, 2006) y la posibilidad de comunicar el conocimiento de forma inmediata, abierta y masiva a través de la red, nos llevan a pensar en la promesa de la transformación de la ciencia y de la apertura de los procesos de investigación. En los últimos treinta años de convivencia con la web, hemos aludido a diversos cambios de paradigma en la conformación de una nueva sociedad digital (Méndez, 2003), sin embargo, continuamos realizando, publicando, financiando y evaluando la investigación de la misma forma que en el siglo pasado.

La Open Science, ciencia abierta o ciencia en abierto implica un cambio radical en la forma de hacer investigación y de difundir los resultados, donde se combina un gran desarrollo tecnológico y un cambio cultural hacia la apertura y la colaboración, promoviendo una ciencia más eficiente, transparente y accesible. La colaboración y la difusión inmediata de resultados de investigación (que son los elementos fundamentales de la Open Science) son aspectos lógicos en el mundo digital en que se produce y difunde la investigación. En este ensayo se recogen las reflexiones, acciones, desafíos y recomendaciones realizados en el contexto europeo en el ámbito de la comunicación científica, con el deseo de devolver la ciencia a la sociedad que la sufraga. No obstante, se critica la proliferación de recomendaciones y se propone entender la ciencia abierta como una actitud, no un movimiento y como un proceso, no un objetivo. Se plantea la necesidad de gestionar un cambio complejo, donde confluyan las políticas científicas y la práctica de los agentes implicados en un escenario global, donde el caso de la COVID-19 subraya este planteamiento y esta necesidad de una ciencia abierta, transparente, generosa y global.

\section{Open Science. Más allá del Open Access: Open Knowledge}

\subsection{Concepto y alcance de la Open Science en un contexto de investigación e innovación responsables}

Open Science es la expresión con la que designamos la práctica de compartir el conocimiento resultante de la investigación financiada con fondos públicos, de forma completamente abierta, libre y sin restricciones de uso. Hay casi tantas definiciones de OS, como autores hablamos de ella y existen diversos análisis de revisión bibliográfica que recogen esta circunstancia (Vicente-Saez y Martinez-Fuentes, 2018), que permiten incluso hablar de escuelas o tendencias para determinar el concepto (Fecher y Friesike, 2014).

Ante la variedad de definiciones, en una de las sesiones de trabajo de la European Open Science Policy Platform (OSPP) ${ }^{1}$ consultamos a los miembros, a través de una herramienta de votación online sobre qué elementos o significados tenían para ellos la Open Science. Surgieron descriptores como: movimiento, actitud, compromiso, reutilización, co-creación y transparencia; y entre los elementos más importantes: acceso abierto, datos abiertos, ciencia ciudadana, pero también, métodos abiertos, innovación abierta, nuevas destrezas ( por ejemplo en la gestión de datos) y aparece de nuevo la transparencia, la diversidad y la inclusión, que nos lleva a pensar en la importancia de la ciencia abierta en el contexto del objetivo 10 de los Objetivos de Desarrollo Sostenible (ODS) ${ }^{2}$. La figura 1 refleja los resultados de la discusión, donde llegamos al acuerdo que la Open Science tenía como común denominador la apertura de los resultados de investigación, no sólo publicaciones (Open Access) y datos (Open Data), sino también otros resultados, métodos, software, etc. Asimismo, se subrayó la colaboración, no sólo como una práctica científica en la nueva forma de hacer investigación, sino también en el sentido de la necesidad de colaboración entre los distintos agentes implicados en el proceso científico para que la ciencia en abierto sea una realidad.

Fruto de estas reflexiones, definimos Open Science como la «investigación académica que es colaborativa, transparente y reproducible, cuyos resultados es-

1 La OSPP, Open Science Policy Platform, o Plataforma Política para la Open Science es un grupo de veinticinco expertos, que representan a diferentes grupos paneuropeos de agentes implicados en la Open Science. Este grupo se concibió como un grupo de alto nivel para asesorar a la Comisión Europea, y en particular al Comisario Carlos Moedas, a lo largo de dos mandatos de la plataforma (2016-2020). La composición de los miembros se dio a conocer en el Consejo de Competitividad de mayo 2016. Se puede consultar un listado actualizado a 19/11/2019 de los miembros por grupo -stakeholders- al que pertenecen, incluyendo sus biografías en: https://ec.europa.eu/research/ openscience/pdf/ec_rtd_ospp-nominated-members_2019.pdf\#view=fit\&pagemode=none

2 ODS-10: https://www.un.org/sustainabledevelopment/es/inequality 
Figura 1. Descriptores y elementos constitutivos de la Open Science (Consulta a los miembros de la EU-OSPP)

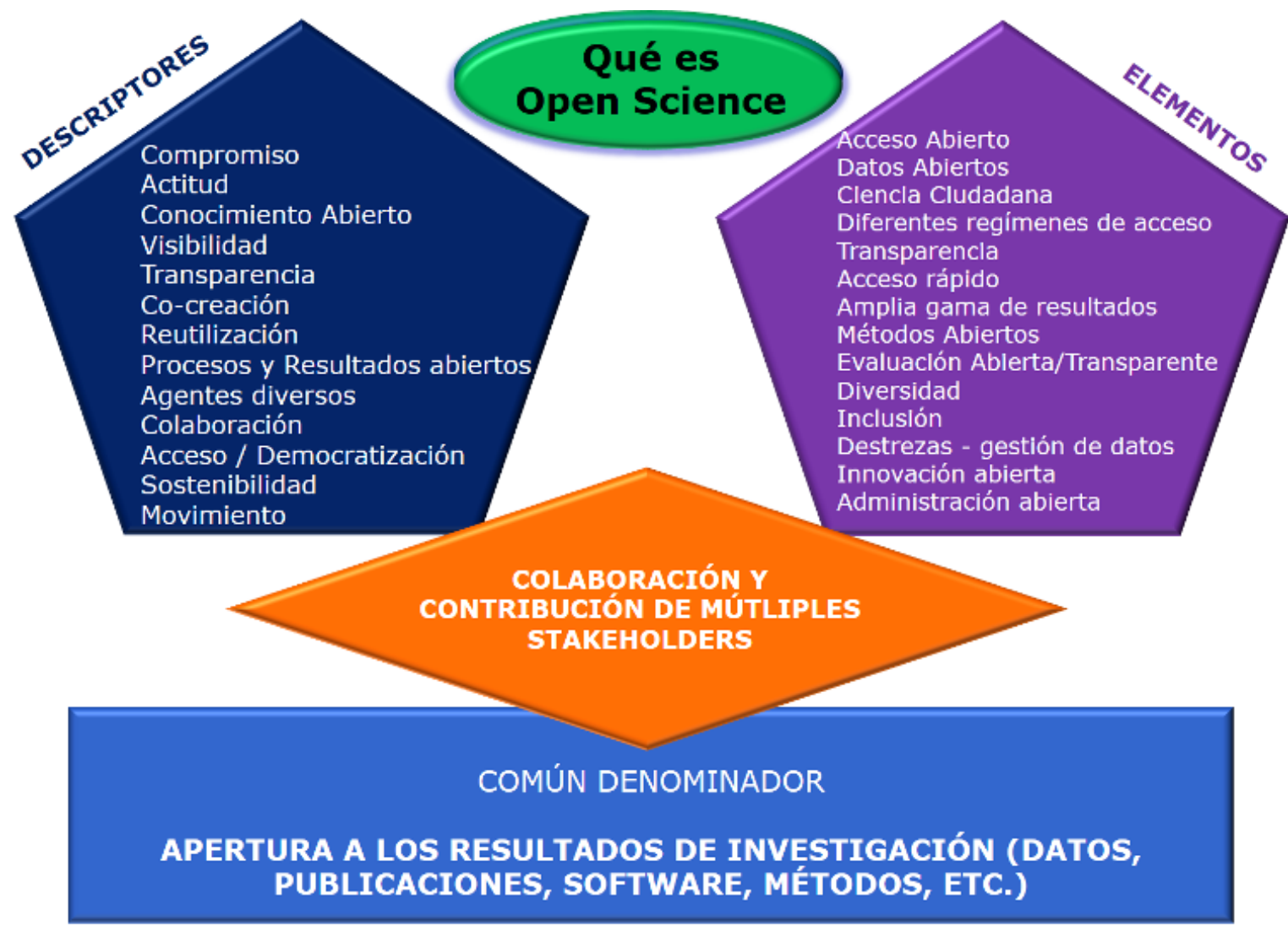

Adaptación de la figura presentada por Michella Bertero y Eva Méndez en la reunión de marzo 2017 de la OSPP

tán públicamente disponibles» (OSPP-REC 2018). Con esta sencilla definición influimos la acepción de ciencia abierta de la Comisión Europea $(2019)^{3}$, que destaca un "cambio sistémico» que permite mejorar la ciencia a través de formas colaborativas y abiertas de producir y compartir datos y conocimiento, tan pronto como sea posible, en el proceso de investigación. No obstante, algunas de las definiciones más citadas, discutidas y refrendadas, definen la Open Science como una idea, un esfuerzo, una forma o una práctica. Así,

"... es la idea de que el conocimiento científico de todo tipo debe compartirse abiertamente tan pronto como sea prácticamente posible en el proceso de descubrimiento» (definición informal de la Open Science de Michael Nielsen en Gezelter, 2011).

«... se refiere habitualmente a los esfuerzos para hacer que los resultados de la investigación financia- da con fondos públicos sean más accesibles en formato digital para la comunidad científica, el sector empresarial o la sociedad en general ... para promover la investigación a largo plazo, así como la innovación» (OECD, 2015).

"... es la Ciencia que se lleva a cabo y se comunica de tal forma que permite a otros contribuir, colaborar y sumarse al esfuerzo de investigación, con todo tipo de datos, resultados y protocolos disponibles, de forma gratuita, en las diferentes etapas del proceso de investigación» (Research and Information Network, 2010).

«... la práctica de la ciencia de tal forma que otros pueden colaborar y contribuir, donde los datos de investigación, las notas de laboratorio y otros procesos de investigación están disponibles libremente, bajo términos que permiten la reutilización, redistribución y reproducción, tanto de la investigación en sí misma,

3 Open Science Fact Sheet (actualizada en diciembre 2019). https://ec.europa.eu/info/sites/info/files/research_and_innovation/knowledge_publications_tools_and_data/documents/ec_rtd_factsheet-open-science_2019.pdf 
como de los datos y métodos subyacentes» (Bezjak et al., 2018; FOSTER project 2015)4.

De todas estas definiciones, yo suscribo las que entienden la OS como una práctica. Vicente-Saez y Martínez-Fuentes, en su revisión bibliográfica (2018) establecen que el término ciencia abierta se refiere, de forma general, a la creación científica de conocimiento transparente y accesible que se comparte y desarrolla a través de redes colaborativas. Sin embargo, desde mi punto de vista, el objetivo de la ciencia abierta no debe centrarse sólo en la apertura de la creación científica per se, sino en la apertura del ciclo de la investigación, en sus distintas etapas, y hacer que los resultados sean más reproducibles, además de abordar retos sociales «con y para la sociedad» ${ }^{5}$. Esa es para mí la clave: el impacto social. En este sentido es importante destacar también la colaboración y la participación, incluso destacar el fenómeno de la ciencia dirigida por los usuarios (user-led science) que concurre en la Open Science, como una forma de abordar, más de cerca, las necesidades de los usuarios (Stodden, 2010) los pacientes o los ciudadanos.

Son muchos los autores además que usan Open Science como un término paraguas que abarca múltiples asunciones sobre el futuro de la creación y diseminación del conocimiento (Fecher y Friesike, 2014). Además, se han generado diversas representaciones de los elementos que recoge el paraguas de la Open Science. Blogs, ponencias, slides, informes, hacen referencia a esta metáfora al definirla. Por ejemplo, una de las primeras representaciones como paraguas fue la que realizó en 2013, un autor coreano en Flickr $r^{6}$ y que el proyecto FOSTER difundió en múltiples de sus recursos ${ }^{7}$. Posteriormente lo hizo el vídeo del proyecto Ubatuba en Brasil ${ }^{8}$, y la bibliotecaria Robin Champieux cuya imagen es utilizada para ilustrar múltiples blogs sobre ciencia en abierto ${ }^{9}$. Más recientemente, la representación de Raluca Blujdea $(2020)^{10}$ pone bajo el paraguas los badges del Center for Open Science $(\mathrm{COS})^{11}$ para explicar el proyecto RepoducibiliTea. El paraguas es también la representación metafórica que ha elegido el informe de Comunidad de Prácticas de Ciencia ciudadana y Open Science de la Citizen Science Global Partnership (CSGP) que pone bajo el de la Open Science los tres elementos clave de la Ciencia Abierta (ver 3. Elementos, desafíos y recomendaciones para la Open Science en Europa), que suscribo completamente: ciencia ciudadana, datos abiertos y publicaciones abiertas (Wehn et al., 2020).

Incluso yo misma dibujé mi propio paraguas de la Open Science, presentado por primera vez en el I/ Encuentro de Ciencia ciudadana celebrado en Medialab Prado (Madrid) en Noviembre de $2016^{12}$ y posteriormente en otras ponencias ${ }^{13}$. Sin embargo, mi metáfora necesitaba algo que tuviera raíces, además de un entorno o una base donde desarrollarse y crecer. Así dibujé Open Science Mushroom (Fig.2) que se ha convertido en un icono, divertido y expresivo, de las implicaciones de la ciencia en abierto ${ }^{14}$, donde la RRI (Responsible Research and Innovation) es el terreno

4 FOSTER project. Open Science Definition: https://www.fosteropenscience.eu/foster-taxonomy/open-science-definition

5 Se utilizan deliberadamente las comillas porque ese es el eslogan del programa SWAFS de Horizonte 2020: https://ec.europa.eu/programmes/horizon2020/en/h2020-section/science-and-society

6 지우 황 (2013): https://flic.kr/p/gxKYeW

7 https://www.fosteropenscience.eu

8 UBATUBA Open Science en Brasil (2017) https://www.youtube.com/watch?v=xGHUhl1WB9E\&feature=youtu.be\&list=PLA9qHoDitstqENOIYLdWy-r7JZbRXC c2\&t=126

9 Kirstie Whitaker (2018) https://whitakerlab.github.io/blog/Open-Science-Blog-Post

10 https://vusci.blog/2020/04/17/reproducibilitea-blends-and-flavors

11 https://www.cos.io/our-services/badges

12 II Encuentro de Ciencia Ciudadana. 26/11/2016. (Vídeo): https://youtu.be/vIDK14a9IX8?t=1716

13 Research Data in an Open Science World. Presentado en LEARN workshop, London, 5 May 2017:https://www.slideshare.net/learnRDM/ research-data-in-an-open-science-world-prof-dr-eva-mendez-uc3m

14 A pesar de que hasta ahora esta representación no se había incluido formalmente en ninguna publicación esta imagen ha sido ampliamente citada en la bibliografía sobre ciencia en abierto (Ochoa-Gutiérrez y Uribe-Tirado 2018), y sobre todo, en redes sociales: @MichellaBertero (marzo 2017): https://twitter.com/evamen/status/844158610225664000?lang=en; @martateperek y @simonhodson99 (mayo 2017): https://twitter.com/martateperek/status/860436638060687360?lang=en; @eoscpilot (lanzamiento de EOSC, noviembre 2018): https://twitter.com/eoscpilot/status/1065910129697144832; @siobhansleeman (marzo 2019): https://witter.com/ siobhansleeman/status/1103991967111876608/ y @NoeleenMcDonald (marzo 2019): https://twitter.com/NoeleenMcDonald/status/1104066703401631744 @etothczifra (marzo 2019): https://twitter.com/etothczifra/status/1107941688234917888; @lluisanglada (junio 2020): https://twitter.com/lluisanglada/status/1270721885320310789 Esta representación ha dado incluso lugar a nuevas versiones, sobre todo en el ámbito de la sinergia de Open Science - Citizen Science (@carnepaoneto (abril 2020): https://twitter.com/ maballesrin/status/1245767090642006018 
Figura 2. Imagen original de la autora.

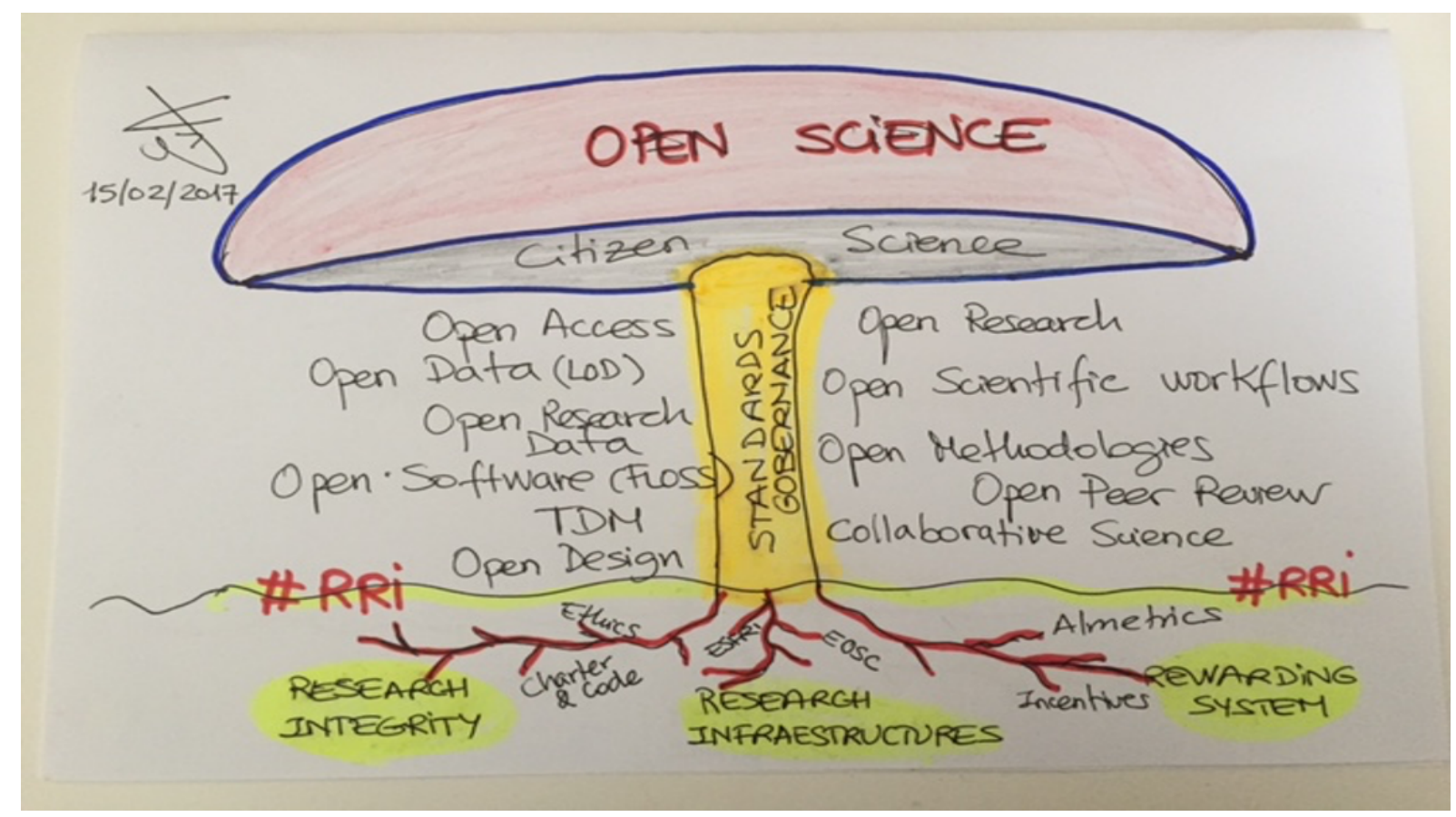

CC-BY Eva Méndez

de fundamentación ética y filosófica que ha permitido creer en la Open Science; la ciencia ciudadana son las esporas, que permiten a la ciencia cuestionarse y reproducirse, mientras las raíces, que deben cimentar el sistema de ciencia en abierto, son las infraestructuras de investigación (incluyendo elnfraestructuras), la integridad científica y un nuevo sistema de incentivos.

La Investigación e Innovación Responsables ${ }^{15}$ (RRI) implica un planteamiento filosófico y de largo alcance ético, que evalúa las expectativas de la sociedad con respecto a la investigación y la innovación, con el objetivo de fomentar el diseño de la investigación y la innovación inclusivas y sostenibles. La RRI incluye el Open Access como un elemento, junto a cuestiones éticas, igualdad de género, participación ciudadana, educación científica y gobernanza del proceso científico e innovador. Bernd C. Stahl define la RRI como una responsabilidad o meta-responsabilidad de alto nivel que tiene como objetivo dar forma, mantener, desarrollar, coordinar y alinear los procesos, actores y responsabilidades relacionados con la investigación y la innovación, de tal forma que se puedan garantizar los resultados de investigación deseables y aceptables (Stahl, 2013). En su definición de RRI, René von Schomberg (2012;
2019) incide en la relación entre los agentes en la innovación, que tiene que ser transparente e interactiva y con una receptividad mutua. Los principios éticos y la meta-responsabilidad de la RRI incluyen el acceso abierto al conocimiento, pero envuelven además todos los procesos y desafíos de la Open Science. El concepto de RRI se ha utilizado para describir una nueva forma de regir la investigación y las relaciones entre los agentes implicados, lo que se denomina cuádruple hélice cuando hablamos de innovación: academia, industria, gobierno y sociedad civil.

La RRI ha sido clave en la política científica europea en Horizonte 2020, y lo seguirá siendo sin duda en Horizonte Europa, como se refleja en el último informe del Programa SWAFS (Delaney et al., 2020) donde ya se recoge la Open Science (no Open Access), como un elemento fundamental de la RRI.

\subsection{Orígenes de la ciencia abierta y Open Science como marca en el mundo del conocimiento abierto.}

De igual forma que con la definición, y como ocure con casi cualquier nuevo concepto, también hay bastantes teorías sobre los orígenes de la Open Science (Bücheler y Sieg, 2011; David, 2008 y 2014) que

15 https://ec.europa.eu/programmes/horizon2020/en/h2020-section/responsible-research-innovation 
surgen en el ámbito de la historia económica y la sociología de la ciencia y ponen el acento de apertura, en la dimensión económica del conocimiento y en el capitalismo intelectual de finales del siglo XVII. Desde la sociología de la ciencia, el principio de la apertura se ha visto como algo consubstancial de la actividad académica (Merton, 1974). La filosofía mertoniana describe la ciencia como un proceso relativamente autónomo, que descansa en el reconocimiento a la primera persona que contribuye con un nuevo descubrimiento científico, a quien le otorga un mérito.

Sin embargo, la denominación Open Science, como tal, se decide y se instala en Europa después de un detallado proceso de consulta realizado por la Comisión Europea entre 2014-2015 y refrendado por los agentes implicados y los Estados miembros (Burgelman et al., 2019). En primer lugar se realizó una consulta pública (julio-septiembre 2014), después hubo seminarios y talleres de validación (octubre-diciembre-2014); el proceso concluyó en febrero de 2015 con un informe final de validación donde se legitimaba, de forma preferente, la denominación de Open Science frente a otras denominaciones como: Science 2.0 o Science in transition (European Commission, 2015a). El apoyo de los Estados miembros llegó en mayo de 2015 a través del debate político mantenido por el Consejo de Competitividad sobre una economía basada en datos (Council of the European Union 2015). Un apoyo que reflejó además las prioridades o retos de la Comisión incluidas en la estrategia del mercado digital único (European Commission, 2015b).

Durante algún tiempo los términos Open Science y Science 2.0 se utilizaron como sinónimos (Bücheler y Sieg, 2011; Burgelman, Osimo y Bogdanowicz, 2010; Mayer, 2015; Tyfield, 2013; Waldrop, 2008), y algunos autores aún siguen alternándolos (Vignoli, Kraker y Sevault, 2015). Hay también quien, siguiendo la progresión, hablan de Ciencia 3.0 (Albers, Shlonsky y Mildon, 2020; Teif, 2014), o en la irónica reflexión de Frank Miedema (2012) sobre la economía del conocimiento en las ciencias de la vida.

Open Science como concepto, denominación y marca (que es como me gusta verlo a mí), se consagra claramente en Europa en el discurso político del comi- sario Carlos Moedas (2014-2019), en el que la Open Science constituyó uno de los emblemas de su visión de las 3 Oes: Open Innovation, Open Science, Open to the World (European Commission, 2016), apoyado también por los Estados miembros el Consejo de Competitividad de mayo de $2016^{16}$.

Otras formas aceptadas y de uso recurrente para referirnos a la Open Science son Open Scholarship, Open Research y Open Knowledge, denominaciones que pretenden incluir expresamente las artes y humanidades en la ecuación. Varios autores y defensores de la Open Science identificamos con frecuencia Open Science-Open Scholarship (Ayris et al., 2018; Neylon et al., 2019; Tennant, 2020; Tennant et al., 2019; Willinsky, 2005), enfatizando el proceso, la colaboración y la forma de hacer investigación, antes que los propios resultados (Burgelman et al., 2019; von Schomberg, 2019). Open Knowledge, que tiene sin duda mejor traducción al español que Open scholarship, es una forma aún más amplia de referirse a la Open Science, no sólo por incluir todas las disciplinas del conocimiento humano, sino por incorporar también la educación abierta como un elemento de la Open Science, donde mecanismos como MOOCs (Massive Open Online Courses) y otros recursos educativos abiertos (REA) se convierten en nuevas formas de difundir el conocimiento. Open Knowledge es también un nombre propio, porque es la denominación de una fundación, la Open Knowledge Foundation $(\mathrm{OKF}){ }^{17}$ creada en 2004. La OKF es compromisaria de la denominada definición de lo abierto ${ }^{18}$, que implica que cualquiera puede, acceder, utilizar, modificar y compartir libremente para cualquier propósito el conocimiento en todas sus formas (datos, publicaciones, código, etc.). Open Knowledge es también una comunidad creada a raíz del proyecto $\mathrm{COKI}^{19}$, surgido en la Curtin University de Australia, pero que entraña una iniciativa global para medir las universidades como instituciones de conocimiento abierto, incluyendo no sólo Open Access y OS sino también aspectos como diversidad, inclusión y otros, que podríamos considerar como RRI (Montgomery et al. 2018). Y también es la denominación elegida por el grupo de expertos de la Comisión sobre indicadores (Wouters et al., 2019) y por la herramienta Open Knowledge Maps ${ }^{20}$, diseñada

16 https://www.consilium.europa.eu/en/meetings/compet/2016/05/26-27/

17 OKF, Open Knowledge Foundation: https://okfn.org

18 https://opendefinition.org

19 http://openknowledge.community

20 https://openknowledgemaps.org 
por Peter Kraker para aumentar la visibilidad de los resultados de investigación tanto para la ciencia, como para la sociedad.

En la OSPP elegimos subrayar la expresión «conocimiento abierto» por su carácter inclusivo, al mismo tiempo que usamos la marca de Open Science, tanto en las recomendaciones (OSPP-REC, 2018) como en el informe final (Méndez y Lawrence, 2020). A pesar de ser términos intercambiables (Open Science-Knowledge-Scholarship), la bibliografía científica consagra también Open Science: así el buscador científico Lens recoge 2.880 trabajos académicos que tienen esta expresión en el título, mientras que 328 incluyen en el título Open Knowledge, y 200 Open Scholarship ${ }^{21}$. Yo utilizo y difundo la expresión Open Science para contribuir a solidificar el concepto, la marca, y lo hago directamente en inglés, aunque exista traducción al español, para clamar su dimensión global, pero estoy completamente de acuerdo en el alcance necesario del conocimiento abierto.

\section{ELEMENTOS, DESAFÍOS Y RECOMENDACIONES PARA LA OPEN SCIENCE EN EUROPA}

Se ha tratado de definir, desde muchas perspectivas, cuáles son los elementos constitutivos claves de la Open Science, y este es el objetivo de la metáfora del paraguas, y de mi interpretación personal, en evolución, de sus elementos constitutivos reflejados en el apartado anterior. Todos coincidimos en que, además del Open Access a las publicaciones, la Open Science incluye también, y de manera muy significativa, el acceso (abierto) a los datos de investigación, pero no sólo, como a veces se suele simplificar, al ver la Open Science como Open Access+Open Data. Para Masuzzo y Martens (2017) la ciencia abierta se sustenta en cuatro pilares abiertos: datos, artículos, revisiones y código. Los elementos o pilares que conforman la Open Science han evolucionado a través de los años y lo seguirán haciendo de acuerdo al avance de la ciencia y la tecnología. Así, por ejemplo, el proyecto FOSTER creó una taxonomía que ha ido evolucionando e incluye seis elementos en su primer nivel: acceso abierto, datos abiertos, investigación reproducible en abierto, evaluación de la ciencia en abierto, políticas de ciencia abierta, y herramientas para la OS (FOSTER project, 2015 22 ). Sin embargo, en ambas aproximaciones, se omite un elemento fundamental, la ciencia ciudadana, que destacamos al hablar de la metáfora del paraguas en (Wehn et al., 2020).

Desde que comenzamos a trabajar en la European Open Science Policy Platform (\#EUOSPP, OSPP, 20162020), lo hicimos sobre ocho elementos o aspectos fundamentales muy claros, que concretaban, a su vez, las cinco acciones que abanderan la agenda (siempre en borrador) de la Open Science en Europa desde principios el $2016^{23}$. Las cinco acciones políticas tenían y tienen (porque aún siguen vigentes en 2021), un carácter amplio y ambicioso, pero a la vez un espíritu muy claro: 1) el fortalecimiento y la creación de incentivos para la ciencia en abierto; 2) eliminar barreras para la Open Science; 3 ) integrar y promover aún más las políticas de acceso abierto; 4) desarrollar la infraestructura europea de datos y servicios para la ciencia abierta conocida como Open Science Cloud; y 5) la integración de la Open Science en la sociedad para hacer que la ciencia responda mejor a las expectativas sociales y económicas. Estas grandes líneas de acción política se concretan, a su vez en ocho elementos clave o retos que la LERU (Ligue of European Research Universities) (Ayris et al., 2018) denominaría, posteriormente también pilares. Cuatro de esos ocho aspectos, desafíos o pilares tienen que ver con el uso y la gestión de los resultados de investigación (ver 3.1); pero también consideramos otros cuatro aspectos en relación a los actores o agentes implicados (investigadores, instituciones y organismos de financiación) que son, al fin y al cabo, los stakeholders o agentes implicados, representados en la OSPP (ver 3.2). En cualquier caso, es importante destacar que los elementos constitutivos de la Open Science son a su vez los desafíos para su implementación.

\subsection{Retos de la Open Science relacionados con los resultados de investigación}

Los retos que aquí clasificamos como relacionados con los resultados de investigación van más allá del mero hecho de abrir publicaciones y datos. Conllevan acciones más concretas y complejas, tanto desde el punto de vista técnico, como legal y político que dieron lugar a diversos informes, de otros tantos grupos de expertos para analizar en profundidad cada uno de estos retos. Estos informes, de alto nivel y muy exhaustivos, al final no quedaron más que numerosas recomendaciones, a distintos niveles, de no siempre fácil implementación.

21 Búsquedas en LENS: https://www.lens.org (agosto 2020).

22 DOI de la taxonomía (Versión 3) en Fighshare: https://dx.doi.org/10.6084/m9.figshare.1508606.v3

23 https://ec.europa.eu/research/openscience/pdf/draft_european_open_science_agenda.pdf 
a. Datos FAIR (Fíndable, Accessible, Interoperale and Re-usable). Más que abrir los datos de investigación, la Open Science implica compartir datos, que sean localizables, accesibles, interoperables y re-utilizables (Wilkinson et al., 2016). FAIR es un acrónimo (muy afortunado, a mi juicio), que refleja una serie de principios (no estándares), pero que tienen una alta complejidad técnica en relación a metadatos, vocabularios, identificadores persistentes y otros estándares aplicables a los datos. FAIR se extiende a otros resultados de investigación e incluso al software (Lamprecht et al., 2020). Cómo convertir este reto en una realidad, dio lugar a uno de los informes del grupo de expertos en FAIR data (European Commission Expert Group on FAIR Data, 2018) donde se recogen 27 recomendaciones para hacer los datos localizables, accesibles, interoperables y re-utilizables.

Abrir los datos, además de las publicaciones, es un paso más que evoca una mayor transparencia y reproducibilidad de la investigación. Además, la revisión de la directiva europea sobre la reutilización de la información del sector público (Directiva PSI o Directiva de Open Data $)^{24}$ publicada en junio de 2019 incluye los datos de la investigación financiada con fondos públicos, como parte de los datos susceptibles de compartirse en abierto y de reutilizarse lo que fortalece la obligatoriedad de poner estos a disposición no sólo de la investigación, sino también de los ciudadanos. La directiva cita expresamente los principios FAIR y también el principio «tan abierto como sea posible, tan cerrado como sea necesario ${ }^{25}$.

Además de los datos FAIR, otro de los elementos y retos de la OS es la creación de la European Open Science Cloud (EOSC) -recientemente se está denominando también EU Open Science Commons-, una infraestructura europea federada para datos y servicios de investigación, donde todos los investigadores puedan depo- sitar, acceder, analizar y reutilizar datos científicos. La EOSC se presentó oficialmente en Viena en noviembre de $2018^{26}$, pero al ser una infraestructura muy compleja tanto desde el punto de vista técnico como de su gobernanza a través de múltiples proyectos que conforman su ecosistema, está aún en fase de construcción. Para definir este reto, se nombraron dos grupos de expertos que dieron lugar a sendos informes (Mons et al., 2016; Muscella et al., 2018) y a un total de $36(15+21)$ recomendaciones.

b. Métricas e indicadores de nueva generación (Next Generation Metrics, NGM) que permitan reemplazar el sistema de incentivos basado en el factor de impacto de las revistas y en el recuento de citas exclusivamente a publicaciones es el tercer reto vinculado a los resultados de investigación. Este desafío y elemento fundamental busca lo que se ha venido denominando «métricas responsables y métricas alternativas», dio también lugar a dos grupos de expertos y sus dos consiguientes informes (Wilsdon et al., 2017; Wouters et al., 2019), con 25 recomendaciones $(12+13)$ y 149 indicadores potenciales (Wouters et al., 2019) que precisan una implementación real.

c. El ultimo reto en relación a los resultados de investigación es el cambio de formas de comunicación científica y de los modelos de negocio para su financiación. Este reto es el que denominamos "futuro de la comunicación científica» y que dio lugar a otro grupo de trabajo y otro informe con 26 recomendaciones (Guédon et al., 2019). En este sentido, tras la conferencia de EuroScience Open Forum, ESOF en Toulouse ${ }^{27}$, se presentó en septiembre de 2018, el ya famoso Plans ${ }^{28}$ : una acción concreta presentada por el ex director general de Investigación e Innovación de la Comisión Europea, Robert-Jan Smiths junto a diversas agencias de financiación europeas que se comprometían a ponerla en práctica (cOAlition $^{29}$ ), con el apoyo de Science Europe ${ }^{30}$ que las representa. El PlanS implica diez principios, según

24 http://data.europa.eu/eli/dir/2019/1024/oj

25 Texto de la directiva en español (Artículo 10 sobre datos de investigación): https://eur-lex.europa.eu/legal-content/ES/TXT/PDF/?uri=CELEX:32019L1024\&from=EN

26 https://eosc-portal.eu

27 EuroScience Open Forum 2018. Vídeo del debate donde se presenta informalmente el PlanS: https://www.youtube.com/watch?v=TYpFNRIEZWo

28 https://www.scienceeurope.org/wp-content/uploads/2018/09/Plan_S.pdf

29 https://www.coalition-s.org

30 https://www.scienceeurope.org 
los cuales todas las publicaciones resultantes de proyectos financiados con fondos públicos por las agencias de la cOAlitionS deben de publicarse inmediatamente en revistas o plataformas completamente Open Access. La Comisión lanzará oficialmente en marzo de 2021 su propia plataforma de publicación para el acceso abierto inmediato, denominada Open Research Europe (ORE $\left.{ }^{31}\right)$.

\subsection{Retos de la Open Science relacionados con los actores de la investigación}

Los otros cuatro elementos de la Open Science en Europa tienen que ver con los agentes del proceso de investigación e implican elementos constitutivos de la Open Science, pero también retos:

a. Crear una nueva forma de evaluar la carrera investigadora que reconozca e incentive completamente la Open Science. Sin duda alguna, este es el desafío fundamental que afecta diametralmente a los investigadores y que descansa en la adopción de las nuevas métricas que discutíamos en la sección anterior. Sobre este tema se creó también un grupo de expertos, que dio lugar a otro informe más sobre el que trabajamos en la OSPP que, en esta ocasión recoge sólo cuatro recomendaciones pero de gran calado, dirigidas tanto a los creadores de políticas como a las agencias de financiación y empleadores; además de nuevos criterios, recogidos en lo que se conoce como CAM (Career Assessment Matrix) (O'Carroll et al., 2017a) que algunos países como Holanda han comenzado a poner en práctica. ${ }^{32}$

b. Que todos los investigadores, principalmente los más jóvenes, tengan los conocimientos y destrezas necesarias para aplicar la Open Science a sus prácticas de investigación, es otro gran reto que no se puede trivializar. No se puede recriminar que los investigadores no practiquen Open Science, si no tienen los conocimientos necesarios para ponerlos en práctica. La formación en Open Science ha dado lugar a diversos proyectos y acciones para educar a investigadores, bibliotecarios y asistentes de investigación. Uno de los más conocidos es el proyecto FOS-
TER que ya hemos citado así como FOSTER+ que dio lugar a un manual de formación co-creado y multilingüe (Bezjak et al. 2018); pero también iniciativas voluntarias de la comunidad como el Open Science MOOC ${ }^{33}$. La Comisión Europea creó también otro grupo sobre este aspecto capital, que recoge seis recomendaciones, completamente alineadas con el informe de incentivos (O'Carroll et al., 2017b).

c. Una nueva integridad científica y un código ético acordado colectivamente que reconozca la ciencia en abierto como el estándar. En este caso, la Comisión no nombró un nuevo grupo de trabajo específico, sino que adoptó los principios del Código de conducta europeo para la integridad científica (ALLEA, 2017) que reconoce la apertura de publicaciones y datos, así como el incentivo y reconocimiento de prácticas de ciencia reproducible y abierta.

d. El último desafío de la Open Science es, como ya hemos resaltado, de capital importancia: que los ciudadanos puedan contribuir de manera significativa en la investigación y que sean reconocidos como productores de conocimiento en la ciencia europea. Esto es, la ciencia ciudadana que, en sus múltiples dimensiones da cabida a la ciudadanía en el ciclo de la investigación. Este aspecto no dio lugar tampoco a un grupo de expertos, sino que la OSPP trabajó directamente con la European Citizen Science Association, (ECSA) ${ }^{34}$, uno de los agentes representados en la plataforma, para determinar recomendaciones y dirimir prácticas de ciencia ciudadana.

\subsection{Declaraciones, Recomendaciones y meta-reco- mendaciones para Open Science.}

En relación a los ocho retos de la Open Science, los distintos grupos de expertos han emitido un total de 124 recomendaciones, a los que debemos unir los diez principios del Plans, para el acceso a las publicaciones en abierto, los diez principios de la ciencia ciudadana (ECSA 2015) y la matriz de evaluación (CAM) (O'Carroll et al., 2017a)this changed approach must be recognised and rewarded by both employers (when recruiting and promoting researchers.

31 https://open-research-europe.ec.europa.eu/

32 https://www.openscience.nl/files/openscience/2019-02/notitie-erkennen-en-waarderen-van-onderzoekers_en-gb.pdf; Room for Everyone's talent: https://www.vsnu.nl/recognitionandrewards/wp-content/uploads/2019/11/Position-paper-Room-for-everyone\%E2\%80\%99s-talent.pdf

33 https://opensciencemooc.eu

34 https://ecsa.citizen-science.net 
A estas recomendaciones, en la Open Science Policy Platform añadimos nuestras propias indicaciones o meta-recomendaciones (OSPP-REC, 2018), también conocidas internamente como integrated advise ya que recogían sugerencias integradas y priorizadas para cada grupo de actores implicados en el proceso científico, relativos a los ocho retos de la Open Scien$c e$, además de las cinco recomendaciones generales de alto nivel: que cada estado miembro nombrase un coordinador de Open Science, que se garanticen infraestructuras y estándares, que la estrategia de recursos humanos y carreras científicas reconozca la Open Science, la alfabetización en ciencia abierta y la difusión a nivel europeo de las ventajas que para la sociedad tiene la Open Science.

Por otra parte, en el contexto del Grupo de Trabajo de Scholarly Commons de la comunidad FORCE $11^{35}$ se ha realizado en un documento vivo que recoge más de cien declaraciones, manifiestos, afirmaciones y reconocimientos relativos a la Open Science o a alguno de sus aspectos ${ }^{36}$. Todas estas declaraciones sirven para que los diferentes actores en el sistema global de investigación manifiesten su acuerdo con, o refrenden la necesidad de implementar un nuevo paradigma de ciencia en abierto, o en particular alguno de sus elementos (ej. Open Access ${ }^{37}$, Open Data ${ }^{38}$, la evaluación de la investigación y sus indicadores $\left.{ }^{39}\right)$..

La Open Science está repleta de recomendaciones, declaraciones, afirmaciones, principios e incluso meta-recomendaciones, pero aun adolece de una implementación práctica global, real y concreta, a pesar de que el propio trabajo de la OSPP respondió a una de las múltiples Ilamadas a la acción durante la presidencia holandesa del Consejo ${ }^{40}$. Por ello, en el apartado siguiente se analiza la aproximación de la implementación práctica en una confluencia de acciones de políticas y mandatos de aplicación por parte de los agentes implicados.

\section{OPEN SCIENCE EN LA PRÁCTICA ¿TOP-DOWN O BOTTOM UP?}

La teoría, el concepto, los elementos constitutivos, las prioridades, los pilares, el alcance y, hasta las metáforas parecen estar claras, pero lo que realmente sigue siendo la asignatura pendiente es la aplicación real del nuevo paradigma de la ciencia abierta. Es necesario pasar del modo declaración-recomendación al modo implementación, no sólo a nivel político, sino en el nivel individual de los agentes implicados (financiadores, universidades y organismos responsables de investigación, elnfras, asociaciones de investigadores, etc.).

\subsection{Open Science bottom-up. PCls Compromisos Prácticos de Implementación}

En múltiples ocasiones y con diferentes motivos se habla de la percepción de abajo a arriba de la Open Science que apela a la participación de personas y agentes implicados en la práctica y defensa de la ciencia en abierto. Existen más de doscientas treinta comunidades de base sobre Open Science, recogidas en un documento abierto iniciado por Brian Nosek ${ }^{41}$, director ejecutivo del Center for Open Science ${ }^{42}$ y profesor de la Universidad de Virginia. Este listado demuestra que

35 https://www.force11.org/group/scholarly-commons-working-group FORCE11 es una comunidad de investigadores, bibiliotecarios, editores y agentes de financiación de la investigación que tratan de promover el cambio en la comunicación científica moderna a través del uso efectivo de la tecnología.

36 Charters and principles in scholarly communication: http://tinyurl.com/scholcomm-charters la recopilación se ha realizado por Bianca Kramer y Jeroen Bosman de la Unviersidad de Utrech, pero es un documento vivo y colaborativo donde se pueden ir añadiendo declaraciones, etc. relacionadas.

El wiki de OA Directory (OAD) recoge también una lista, menos exhaustiva de declaraciones en: http://oad.simmons.edu/oadwiki/Declarations_in_support_of_OA

37 Las tres declaraciones más importantes sobre Open Access, son las denominadas 3 Bs, Budapest (2002): https://www.budapestopenaccessinitiative.org, Bethesda (2003): http://legacy.earlham.edu/ peters/fos/bethesda.htm y Berlín (2003): https://openaccess.mpg.de/ Berlin-Declaration.

38 Una de las declaraciones más recientes sobre Open Data en la Sorbone Declaration (2020): https://www.leru.org/files/Sorbonne-declaration.pdf promovida por LERU pero refrendada por otras ocho alianzas de universidades en Canadá, Estados Unidos, Alemania, Francia, África y Australia.

39 La declaración más importante en este sentido es la Declaración de San Francisco, DORA, Declaration On Research Assessment (2012) https://sfdora.org, seguido del Leiden Manifesto for Research Metrics (2015): http://www.leidenmanifesto.org, y los más recientes principios de Hong Kong (2019) que también inciden en la integridad de la investigación: https://doi.org/10.31219/osf.io/m9abx

40 Amsterdam Call for Action on Open Science (Abril 2016): https://www.government.nl/documents/reports/2016/04/04/amsterdam-callfor-action-on-open-science

41 A crowd-sourced list of Open Science Grassroots Community: https://docs.google.com/spreadsheets/d/1LNF5_bOkRV-RLIF4HYmu-gOemla4IdfXEer89fM-Vy8/edit\#gid=0

42 https://www.cos.io 
distintas comunidades, instituciones y personas se unieron para apoyar la aceptación y adopción gradual de la Open Science (Vignoli, Kraker y Sevault, 2015). Sin embargo, la implementación debe llegar, más allá del activismo o la defensa colectiva de los principios de la Open Science, a la práctica objetiva tanto en la forma de hacer investigación como en la forma de divulgarla.

En este sentido, el segundo mandato de la OSPP se centró en lo que yo denomino PCls (Practical Commitments for Implementation). Un PCl es un compromiso que una persona, institución, o agente involucrado en el ecosistema de la ciencia moderna adopta para poner en práctica algún aspecto de la Open Science. Los compromisos prácticos de implementación ( $\mathrm{PCl}$ s en sus siglas en inglés) tienen que ser realistas y asequibles, y quien los proponga tiene que tener jurisdicción para adoptarlos; los $\mathrm{PCl}$ responden a la pregunta ¿qué puedo hacer yo para que la Open Science sea una realidad? ${ }^{43}$. Así, por ejemplo, yo veo el PlanS como un $\mathrm{PCl}$ por parte de las agencias de financiación: requieren algo concreto (la publicación inmediata en Open Access de los artículos de proyectos que financian) desde su jurisdicción como sufragadores de los costes de la investigación. El informe final de la OSPP recoge un listado exhaustivo de $\mathrm{PCl}$ por grupo-agente o institución implicada en la plataforma y presentan ejemplos de Open Science desde la práctica de los stakeholders (Méndez et al., 2020).

El informe final de la OSPP es una nueva llamada a la acción, desde la voz de los agentes (bottom-up) para crear un nuevo sistema de investigación. Proponemos una nueva fecha límite (2030), un fundamento (el conocimiento compartido) y cinco atributos: 1) una estructura de carrera académica que reconozca y valore distintos resultados, prácticas y comportamientos que potencien las contribuciones científicas en un sistema de conocimiento compartido; 2) un sistema de investigación que sea confiable, transparente y reconocido -necesitamos confiar en la ciencia-; 3) un sistema que permita la innovación, porque esta mejora la ciencia, la sociedad y progreso tecnológico y aumenta el bienestar; 4) una cultura de investigación que facilite la diversidad, la igualdad y la equidad de oportunidades y que no deje a nadie atrás; y 5) un sistema de investigación que haga políticas basadas en la evidencia. Es fundamental que entendamos que las nuevas formas de trabajar y de hacer ciencia que implica la OS han de estar fundamentadas en la meta-investigación que demuestre que las prácticas existentes que generan disfunciones en el sistema, deben de cambiarse.

\subsection{Implementación top-down: políticas y estrate- gias formales de Open Science}

Además de los compromisos prácticos de implementación, para crear un nuevo sistema de conocimiento compartido, es necesario contar con una política científica sólida que avale y motive su aplicación. Las acciones políticas a nivel de la Comisión son claras, pero también deben de ser coherentes a nivel de los estados miembros de la Unión Europea, tanto desde la regulación, como desde la correcta financiación para hacerlas posibles.

En los últimos cuatro años algunos estados miembros han lanzado sus políticas nacionales concretas para la implementación de la OS: Portugal $(2016)^{44}$, Holanda $(2017)^{45}$, Francia $(2018)^{46}$ y más recientemente, en 2019 , lo han hecho Finlandia ${ }^{47}$, Suiza ${ }^{48}$ e Irlanda ${ }^{49}$. Cada uno de estos países tiene su estrategia: centralizada a nivel gobierno, como en Francia, desde las universidades, como en Suiza, o desde una metodología participativa de co-creación, como en Finlandia. Pero en general todas estas políticas tienen objetivos claros y bien alineados a los retos de la agenda europea: acceso abierto a datos y publicaciones, infraestructuras para el almacenamiento de datos FAIR, así como crear un sistema de incentivos que fomente y motive las prácticas de Open Science, son elementos comunes a todas ellas. Además, Finlandia y Suiza promueven también la educación abierta, e Irlanda pone el acento en la formación en Open Science. La política francesa se caracteriza por el compromiso -clave a mi juicio- de medir el avance hacia una ciencia en abierto a través de indicadores y su monitorización ${ }^{50}$; por reconocer la bibliodiversidad o diversidad de modelos de publicación científica; y por la democratización del

43 Ver Ponencia inaugural en la Open Science Conference (Berlin, Marzo 2019): Open Science?... Darling, we need to talk. https://www. open-science-conference.eu/wp-content/uploads/2019/03/Eva-Mendez.pdf (slide 22).

44 https://www.ciencia-aberta.pt

45 https://www.openscience.nl

46 https://www.ouvrirlascience.fr/open-science

47 https://openscience.fi

48 https://www.swissuniversities.ch/fileadmin/swissuniversities/Dokumente/Organisation/SUK-P/SUK_P-2/OpenScience_Strategy_v2.5_clean.pdf

49 http://norf-ireland.net

50 https://ministeresuprecherche.github.io/bso 
conocimiento y la ciencia participativa (Ministère de L'Enseignement Supérieur de la Recherche et de L'Innovation, 2020).

En España no contamos todavía con una estrategia de Open Science, aunque sí de Open Access. Desde 2011 la Ley de la Ciencia la Tecnología y la Innovación ${ }^{51}$ establece el acceso abierto a las publicaciones financiadas con proyectos del Plan Estatal de Investigación. Se ha creado además una Comisión de Open Science (COS) coordinada por la Fundación Española para la Ciencia y la Tecnología (FECYT) para establecer las bases de una estrategia nacional de Open Science. Asimismo, la Estrategia Española de Ciencia, Tecnología e Innovación 2021-2027, ve la ciencia abierta "como una oportunidad que permitirá mejorar la calidad, la transparencia, el impacto, la reproducibilidad y el acceso de la ciudadanía al conocimiento»; y vincula ciencia abierta y excelente, donde la Open Science favorecerá la generación de conocimiento de alta calidad e impacto (Gobierno de España. Ministerio de Ciencia e Innovación 2020). Las universidades españolas, a través de su conferencia de rectores (CRUE) también mostraron en febrero de 2019 su compromiso para implantar la Open Science ${ }^{52}$.

Los gobiernos, y sus políticas científicas y de financiación de la investigación, tanto a nivel nacional, regional y europeo (top-down), tienen un valor decisivo a la hora de actualizar procedimientos, renovar sistemas de evaluación y crear incentivos y marcos regulatorios que potencien la Open Science y el cambio cultural, necesario y complejo, que esta implica. Asimismo, las comunidades y quienes investigamos (bottom-up) somos fundamentales para dar forma a la implementación real de la Open Science, desde una visión realista en sus disciplinas, centros de investigación y flujos de trabajo.

\section{DEL MOVIMIENTO OPEN A LA GESTIÓN DE UN CAMBIO COMPLEJO}

\subsection{Open Science: pleonasmo, paradigma, derecho y actitud}

En la wikipedia, que es, de forma realista hoy en día, el punto de inicio en el que el lector puede encontrarse una definición, y por tanto una de las definiciones más difundidas, se clasifica a la Open Science como un movimiento. Así, se ha adoptado esa caracterización en distintos ámbitos (UNESCO) ${ }^{53}$ y por diversos autores (Leonelli, Spichtinger, y Prainsack 2015; Nielsen 2011a y 2011b; Roman, Liu y Nyberg, 2018), que refieren en algún momento a la Open Science como movimiento, asimilándolo en algunos casos, a otras tendencias open que concurren en el contexto de la Open Science: source, access, data (Willinsky, 2005), open educational resources, open pedagogy, open course development (Jhangiani y Biswas-Diener, 2017) e incluso open standards y open design (Pomerantz y Peek, 2016). Sin embargo, a mí no me gusta hablar de movimiento ya que evoca una aproximación demasiado bottom-up de la Open Science (ver 4.1.) y reviste el concepto de un mero activismo cuando, en realidad, la Open Science viene de la mano de la militancia en el conocimiento abierto y de un nuevo ethos que transciende la discusión binaria de abierto-cerrado, hablando sólo de resultados de investigación (o, aún peor, sólo de las publicaciones).

Para mí, la expresión Open Science es, en primer lugar, desde un punto de vista lingüístico, un pleonasmo, ya que la apertura debería de ser consubstancial a la ciencia, y por lo tanto abierta es un vocablo innecesario, redundante, para que tenga su sentido. Sin embargo, hoy en día necesita de ese adjetivo para añadirle expresividad ${ }^{54}$. Llegará un momento en que abierta sea la situación por defecto, la nueva normalidad, como evoca el título de este ensayo, y sólo hablemos de ciencia.

En este sentido, me gusta hablar del conocimiento abierto como un paradigma para la ciencia, que tiene una marca para denominarlo (Open Science). La Open Science implica un cambio de paradigma en el sentido más amplio que Thomas Khun utilizó en su Estructura de las revoluciones científicas al analizar el comportamiento de las comunidades científicas encargadas de seleccionar la forma más apta de practicar la ciencia en el futuro. En la investigación, cuando un paradigma deja de dar respuesta al problema que analiza, se producen disrupciones a nivel científico y se crean otros nuevos (Kuhn, 2012). En la forma de comunicar esa investigación ocurre lo mismo, el paradigma no funciona: es un paradigma creado en el siglo XVII, basado exclusivamente en las publicaciones y en el impacto

51 Ley 14/2011, de 1 de junio, de la Ciencia, la Tecnología y la Innovación: https://www.boe.es/eli/es/l/2011/06/01/14/con

52 http://www.crue.org/Documentos\%20compartidos/Informes\%20y\%20Posicionamientos/2019.02.20-Compromisos\%20CRUE_OPENSCIENCE\%20VF.pdf

$53 \mathrm{http}: / /$ www.unesco.org/new/en/communication-and-information/portals-and-platforms/goap/open-science-movement

54 https://dle.rae.es/pleonasmo 
de las revistas en las que se publica; está lleno de términos anacrónicos y espurios en el contexto de la edición electrónica (paper, manuscrito, pre-print, página, etc.); está repleto de disfunciones y no responde ni a la realidad del momento tecnológico, ni a las necesidades de la sociedad.

Por eso hay que crear otro paradigma nuevo, coherente con las posibilidades y necesidades actuales. La propia noción de paradigma tiene un carácter de fundamento y de modelo organizador y suministrador de una orientación epistemológica, pero también creadora de modelos. En este sentido la Open Sciece, por un lado, añade un cuarto modelo al propio proceso de investigación tradicionalmente basado en tres paradigmas (teórico, experimental y observacional), al que ahora se suma el de la computación masiva de datos. Y por otro lado, la Open Science añade una nueva forma de comunicar la investigación: «de-construir el proceso de comunicación científica utilizado en el paradigma impreso y reconstruirlo de forma que tenga sentido en la Web» (National Research Council, 2012). Por supuesto, no soy la única que ve la Open Science como un paradigma, de hecho, la denominación de Ciencia 2.0, es a mi juicio, una alusión tácita a la necesidad de una nueva versión del sistema científico, tanto en la forma de hacer investigación como de comunicarla y evaluarla, y quizás alude también a la «nueva revolución» que reclama Neilsen (2011a).

La declaración universal de los Derechos Humanos establece en su artículo 27 el derecho fundamental a la ciencia y la cultura: "Toda persona tiene derecho a tomar parte libremente en la vida cultural de la comunidad, a gozar de las artes y a participar en el progreso científico y en los beneficios que de él resulten» (United Nations 1948). En 1966 en el Pacto de Derechos Económicos, Sociales y Culturales ${ }^{55} \mathrm{se}$ amplió este reconocimiento y se incluyó el «disfrute» del progreso científico. No obstante, aunque se ha reconocido y estudiado (Shaver, 2009) el derecho a los beneficios y resultados de la ciencia, y a diferencia de otros derechos humanos, no está legalmente definido ni articulado cómo acceder a él (Wyndham y Vitullo, 2018). Por ello desde $2018^{56}$ Naciones Unidas ha retomado esta cuestión y, a través del Comité de Derechos Económicos, Sociales y Culturales ${ }^{57}$ (2020), se está debatiendo este derecho para asegurar que los ciudadanos puedan beneficiarse de la ciencia y, si quieren, participar en ella. El derecho fundamental a la ciencia y a sus resultados pone en evidencia el pleonasmo de la ciencia abierta. Más recientemente y con motivo de la situación generada por la COVID-19 se ha vuelto a destacar este aspecto. Capps (2020) sostiene que los bienes comunes abiertos generan una comunidad de derechos, formada por personas e instituciones, cuyos intereses apoyan mutuamente el bien público. $\mathrm{Si}$ la ciencia abierta -aquí el pleonasmo- es un derecho y una piedra angular del estado de bienestar, entonces debería reafirmarse como bien público esencial y como derecho fundamental.

Por último, en esta caracterización particular de la Open Science, me gustaría destacar que es, sobre todo, una actitud, más que un movimiento, en el sentido de que su implementación implica una forma de operar y de relacionarse con los agentes y con los resultados del sistema de investigación, que evoca una nueva conducta necesaria.

\subsection{La gestión de un cambio (de paradigma / cultu- ral) complejo}

Se han hecho múltiples estudios y encuestas a los investigadores de distintos niveles (O'Carroll, et al., 2017a; Heise y Pearce, 2020; Scheliga y Friesike, 2014) incluyendo investigadores nóveles (Allen y Mehler, 2019) y distintas latitudes (Beaudry et al., 2019; Pardo Martínez y Poveda, 2018), para detectar cuál es su conocimiento sobre la Open Science y analizar los principales problemas para ponerla en práctica. También se han analizado en este sentido, instituciones como universidades y agencias de financiación, fundamentalmente en relación a sus políticas de incentivos y/o financiación de la investigación (O’Carroll et al., 2017a; Saenen et al., 2019) e incluso el sector privado (Laursen y Salter, 2014; Simeth y Raffo, 2013). Todos coinciden/coincidimos en que la mayor barrera para la ciencia en abierto es el sistema tradicional de incentivos que se basa casi exclusivamente en el factor de impacto, con el inconveniente de las revistas con impacto más alto suelen ser las que se encuentran tras barreras de pago y modelos anacrónicos de suscripción, muy rentables para las grandes editoriales pero que llevan, en el mejor de los casos, a pagar con fondos públicos por el acceso y por la publicación. Algo que

55 https://www.ohchr.org/sp/professionalinterest/pages/cescr.aspx

56 https://www.ohchr.org/EN/HRBodies/CESCR/Pages/Discussion2018.aspx

57 https://www.ohchr.org/EN/HRBodies/CESCR/Pages/DraftGeneralComment_Science.aspx 
el ex-comisario Moedas calificó en varias ocasiones como indignante ${ }^{58}$.

Todo esto hace que se haya establecido algo así como una doble moral en la difusión científica, lo que Scheliga y Friesike (2014) Ilaman «dilema social», donde los investigadores muchas veces nos debatimos entre lo que nos gustaría hacer, en coherencia con las herramientas de producción y la comunicación digital de la ciencia, y con el compromiso de nuestra investigación para la sociedad; y lo que tenemos que hacer, en virtud de lo que se entiende como carrera investigadora. Esta realidad dinamita la riqueza de los retos de la Open Scien$c e, y$ reduce todo el proceso investigador (las preguntas, las respuestas, los implicados, los métodos, las infraestructuras, la colaboración, la transferencia, la innovación, etc.) al deseo ancilar de que me acepte un manuscrito una editorial en una revista que tenga un alto factor de impacto (la revista, no la contribución individual del investigador).

Michael Nielsen admitió en su conferencia TedX (2011) que el fracaso de algunos proyectos de ciencia en abierto se debe a que han recurrido a medios no convencionales para fines convencionales (un ejemplo es el PolyMath Project). La solución pasa por el precepto neoliberal de cambiar los incentivos o, como expresamente dice Nielsen, cambiar la cultura científica, los valores individuales de quienes hacen la ciencia, que entiendan como parte de su trabajo es compartir datos, código e ideas (Nielsen, 2011b).

También hace falta formación, promover el cambio cultual en las instituciones y reconocer que la apertura es una invitación manifiesta a la calidad y a la colaboración. Desmitificar el sistema de calidad de las publicaciones, que nadie se cree, pero que todos los investigadores aceptamos estoicamente. Asimismo, seguir creando nuevas infraestructuras que permitan compartir los resultados de investigación, y también nuevas herramientas para evaluar de forma objetiva y completa el desempeño de cada investigador en un mundo de ciencia abierta, que premie la exposición pública de los resultados de investigación, pero sobre todo su impacto en la sociedad y la capacidad de la ciencia para resolver problemas reales, de manera completamente nueva. Lo difícil es crear un nuevo sistema científico, al mismo tiempo que mantenemos (y pagamos) el anterior.

\section{COVID-19 Y LA URGENCIA DE UNA CIENCIA EN ABIERTO A NIVEL GLOBAL}

La situación de emergencia mundial provocada por la COVID-19 ha puesto en evidencia la urgencia y la globalidad de la ciencia. Este nuevo coronavirus ha atacado no sólo nuestra inmunidad y nuestro statu quo vital, sino también el statu quo del tradicional -y anacrónico- sistema científico y su forma de comunicación, urgiendo la apertura, pero también la excelencia, de la ciencia a nivel global. El espíritu de lo abierto, ha impulsado la respuesta científica a la pandemia (Zastrow, 2020) no sólo desde la publicación de los resultados, sino en las nuevas prácticas de intercambio y acciones colaborativas.

Aunque en este ensayo nos hemos centrado en el entorno europeo, es importante destacar además que la Open Science ha de ser una práctica global, que se base en la misma fundamentación y en los mismos principios en todos los países, a todos los niveles, para todos los investigadores, todas las disciplinas y todos los agentes.

\subsection{No sólo abierta: excelente}

Desde que comenzó la crisis del coronavirus estamos sepultados en una avalancha de estudios científicos ${ }^{59}$ donde asistimos a la investigación en tiempo real o, al menos, en lo que respecta a su publicación (Hocquet, 2020). En este contexto, probablemente sin precedente, son múltiples las reflexiones y acciones para la implementación urgente de la Open Science. Más que nunca se evidencia la metáfora que tanto me gusta mencionar: la ciencia es como un paracaídas: si no se abre, no te va a ayudar. Desde la $\mathrm{OECD}^{60}$ hasta la $\mathrm{UNESCO}^{61}$, pasando por Comisión Europea ${ }^{62}$, se reco-

$58 \mathrm{https}$ ://sciencebusiness.net/framework-programmes/news/free-access-research-papers-2020-impossible-without-radical-steps-sayseu; https://www.aip.org/fyi/2018/european-open-science-agenda-taking-shape

59 https://elpais.com/ciencia/2020-05-04/sepultados-bajo-la-mayor-avalancha-de-estudios-cientificos.html

60 OECD (May 2020): Why Open Science is critical to combatting COVID-19: http://www.oecd.org/coronavirus/policy-responses/why-openscience-is-critical-to-combatting-covid-19-cd6ab2f9/

61 https://en.unesco.org/covid19/sciencesresponse

62 https://ec.europa.eu/info/research-and-innovation/research-area/health-research-and-innovation/coronavirus-research-and-innovation/covid-research-manifesto_en 
noce el aspecto crítico de la apertura para enfrentar y combatir la COVID-19 (Besançon et al., 2020). Se han creado plataformas específicas a todos los niveles ${ }^{63}, y$ hasta los editores comerciales parecen haberse conmovido (o quizás pretenden una estrategia de mercado de contexto) creando centros de acceso libre o gratuito a publicaciones relacionadas con la COVID-1964. En el estudio de Belli et al. (2020) se analizan más de 18.000 publicaciones recogidas en la Web of Science, y determinan que el $90 \%$ están en acceso abierto. Aunque parezca evidente y que no hay marcha atrás (Barbour y Borchert, 2020), es preciso analizar en qué condiciones las editoriales abren esos artículos. Así, en un estudio reciente (Arrizabalaga et al., 2020) hemos analizado 5.611 artículos sobre la COVID-19 en PubMed, y si bien el $97 \%$ están publicados técnicamente en abierto, casi un $70 \%$ de ellos pertenecen a una categoría de acceso abierto bronce, es decir, que las editoriales los abren pero sin dotarles de licencias de reutilización, lo cual implica que, en cualquier momento, podrían volver a cerrar el acceso.

Otras enfermedades como el ébola o el zika ya habían sido buenos ejemplos para demostrar la necesidad de compartir datos y abrir el conocimiento (Burgelman et al., 2019; Chretien, Rivers y Johansson, 2016) y de poner de manifiesto las disfunciones del sistema tradicional de difusión del conocimiento científico al que aludíamos antes. La Organización Mundial de la Salud busca desde 2015 también un cambio de paradigma en la forma de compartir la información en emergencias de salud pública (World Health Organization (WHO) 2015), pero ahora es más evidente que nunca, que se necesita una ciencia abierta y excelente. No obstante, también se ha puesto sobre la mesa la calidad científica de las publicaciones en abierto, reduciendo o trivializando la Open Science a los mecanismos y sistemas de pre-prints (manuscritos sin revisar, subidos por los investigadores antes de su publicación), bioRxiv y medRxiv (los dos repositorios temáticos más importantes relacionados con biología y medicina) que recogen, después de siete meses de pandemia, más de 8.500 pre-prints ${ }^{65}$ sobre la COVID-19. Aunque se ha criticado la baja calidad de muchas de estas publicaciones, los sistemas de pre-publicación han reactivado sus mecanismos de control y han rechazado múltiples manuscritos (Kwon, 2020). Pero también se ha puesto de manifiesto, por un lado, la necesidad de una intermediación cualificada en las nuevas formas de producir investigación basadas en los principios de la Open Science, por ejemplo, por parte de los periodistas ${ }^{66}$. Y por otro lado la correcta utilización de esos principios en una adopción inminente de prácticas de Open Science (como la revisión por pares abierta, los principios éticos en la compartición urgente de datos, etc.), recordando que la ciencia siempre debe ser un proceso riguroso, confiable y transparente, especialmente en el contexto de una pandemia (Besançon et al., 2020).

\subsection{No sólo abierta: global}

La ciencia tiene que ser abierta, pero tiene que serlo a nivel global, no sólo europeo, o de Estados Unidos, o del Norte, o de los países más desarrollados. La transición a la Open Science by design es, de manera inherente, un proceso global (Committee on Toward an Open Science Enterprise et al., 2018). Cuando ponemos la Open Science en la dimensión global, inevitablemente nos podemos hacer preguntas de mayor calado sobre la naturaleza de la ciencia y del conocimiento como las que se hacen los creadores de la OCSDNet (Open and Collaborative Science in Development Network) ${ }^{67}$ al tratar de contextualizar la apertura del conocimiento. Algunas de estas preguntas epistemológicas son: ¿de quién es la ciencia que se abre? ¿quién la abre

63 Algunos ejemplos son: el trabajo colectivo realizado en el contexto de la RDA (Research Data Alliance) para establecer guías y recomendaciones para crear datos FAIR y públicamente accesibles en la medida de lo posible: https://www.rd-alliance.org/group/rda-covid19rda-covid19-omics-rda-covid19-epidemiology-rda-covid19-clinical-rda-covid19-0 La pasarela que ha creado OpenAire para enlazar datos sobre la COVID: https://covid-19.openaire.eu o la comunidad/colección específica en ZENODO, sobre el tema: https://zenodo.org/communities/covid-19 El portal de datos de investigación creado por la Comisión Europea: https://www.covid19dataportal.org La plataforma de publicación de pre-prints Outbreak Science https://outbreaksci.prereview.org El expert finder creado en Australia para buscar e identificar expertos sobre el tema: https://www.science.org.au/covid19/experts Para una lista exhaustiva de recursos sobre la COVID-19, ver: Open Science since COVID-19: Open Access + Open Data: https://osf.io/preprints/socarxiv/a5nqw recopilada por AlejandroUribe-Tirado, et al., en Open Science Framework.

64 Novel Corona Virus Information Center de Elsevier: https://www.elsevier.com/connect/coronavirus-information-center o Coronavirus (COVID-19) Research Highlights creado por Springer Nature: https://www.springernature.com/gp/researchers/campaigns/coronavirus

65 https://connect.biorxiv.org/relate/content/181

66 Debate UPNa: Juan Ignacio Pérez Iglesias; Pampa García Molina; Eva M. Méndez Rodríguez, El desbordamiento del Sistema de publicaciones cientificas en: https://www.youtube.com/watch?v=IzFq-Sy2d2I

67 https://ocsdnet.or 
o quién la tiene que abrir? ¿quién se va a beneficiar de estos nuevos marcos y prácticas? ¿cuáles son los riesgos? ¿llevará esto a la igualdad y equidad en el acceso y la producción de conocimientos por parte de investigadores en entornos desiguales? ¿alterará la Open Science la estructura de poder global existente para la legitimación del conocimiento? ¿conducirá a una mayor marginación del conocimiento del Sur Global? ¿cómo contribuirá a los Objetivos de Desarrollo Sostenible (ODS)? (Chan, 2019).

Que la Open Science sea una práctica a nivel mundial es clave para garantizar la equidad desde el punto de vista del derecho humano a la ciencia, pero también desde el punto de vista funcional y de la coherencia de un sistema de conocimiento compartido global. La COVID-19 instigó la acción de 122 países en el reconocimiento de la Open Science ${ }^{68}$ y también produjo la declaración de los ministros de ciencia del $\mathrm{G}^{69}$. Pero aparte de este efecto catalizador del coronavirus, el G7 reconoce la importancia de la OS global desde 2017 cuando creó un grupo de trabajo sobre Open Science. Asimismo, Naciones Unidas convocó en noviembre de 2019 la conferencia Hacia una Ciencia Abierta Global ${ }^{70}$, donde se reconoció a la Open Science como un elemento clave para la Agenda 2030 y los ODS. La UNESCO está en pleno proceso de creación de una Recomendación de Open Science ${ }^{71}$ que solicitaron 193 países y que será adoptada en 2021. La OS sólo puede cambiar la empresa global de la ciencia si la comunidad científica internacional adopta sus prácticas y principios. Tiene el potencial, además, para la UNESCO, de mejorar los enfoques de la investigación e innovación responsables (RRI) y brindar transparencia en todo el sistema de ciencia, tecnología e innovación.

\section{A MODO DE CONCLUSIÓN}

A pesar de que el Open Access ya es mayor de edad (18 años desde la declaración de Budapest) y que llevamos, al menos cinco años, desde que adoptamos la Open Science como marca y objetivo, todavía estamos lejos de llegar a un cambio de paradigma completo y necesario y a una nueva forma de hacer y comunicar la investigación. La ciencia abierta va mucho más allá del acceso abierto a las publicaciones, y también mucho más allá que el acceso abierto a los datos de investigación, como se ha discutido a lo largo de este trabajo. Se resumen a continuación algunas de las conclusiones a las que ha llegado la autora y que se han apuntado a lo largo del texto:

- La Open Science (particularmente en Europa) está repleta de recomendaciones, declaraciones, afirmaciones e incluso de meta-recomendaciones. Es preciso una mayor definición de lo que yo llamo compromisos prácticos de implementación (PCls), de todos los agentes implicados.

- Open Science, Open Scholarship y Open Knowledge, se pueden utilizar como sinónimos y normalmente el uso de una u otra denominación varía en contextos y latitudes. Ninguno de estos términos alude, a mi juicio, a un movimiento, sino a una actitud y a una forma de hacer investigación que enfatiza los procesos y la colaboración, no los resultados. Sin embargo, yo defiendo la denominación de Open Science como marca global para denominar el nuevo paradigma que clama la investigación moderna.

- La Open Science no sólo nos proporciona herramientas y nos pone frente a retos, también implica una oportunidad excepcional de revisar el sistema científico actual, reflexionar sobre su anacronismo y adaptar la ciencia al contexto presente: digital, colaborativo y basado en datos.

- El cambio de paradigma real, la República de la Open Science, se producirá en la confluencia de políticas (top-down) y compromisos prácticos de implementación (PCls, bottom-up); ambas aproximaciones son necesarias para que se produzca el cambio cultural necesario.

- Necesitamos una nueva revolución científica, reinventar el descubrimiento que decía Nielsen, para romper el estancamiento de la ciencia moderna en la comunicación científica del siglo pasado. Que la ciencia sea capaz, no sólo de responder preguntas y de crear papers, sino de resolver problemas.

- El sistema científico actual es disfuncional y está en transición. Se enfrenta a desafíos cada vez mayores relacionados con la confianza en la ciencia, el derecho a la ciencia y la reproducibi-

\footnotetext{
68 https://www.researchprofessionalnews.com/rr-news-world-2020-4-coronavirus-catalyses-global-action-on-open-research

$69 \mathrm{https://www.state.gov/g7-science-and-technology-ministers-declaration-on-covid-19}$

70 https://research.un.org/conferences/OpenScienceUN

71 https://en.unesco.org/science-sustainable-future/open-science/recommendation
} 
lidad, y necesita ser más abierto, colaborativo y eficaz.

- La reciente pandemia del coronavirus ha demostrado que la ciencia tiene que ser abierta y global. En una emergencia, los datos y otros resultados son cruciales para la investigación colaborativa y la toma de decisiones.

A nivel europeo, el nuevo programa marco (Horizonte Europa) reabrirá en 2021 muchos de los objetivos de la Open Science en los países de la Unión Europea. Esperemos que no solo se cambie la fecha del horizonte, sino que se alcance el objetivo, y que la Open Science sea, para la investigación, la nueva normalidad que nos ha impuesto la COVID-19 para el resto de aspectos de nuestras vidas.

\section{AGRADECIMIENTOS}

Quiero expresar mi agradecimiento a muchos colegas con quienes he mantenido largas discusiones en los últimos cinco años, que han alimentado, sin duda, las tesis y reflexiones sobre Open Science que recojo en este artículo: Rebecca Lawrence, Michella Bertero, Jean-Claude Burgelman, Catriona McCallum, Barend Mons, Karel Leuven, Natalia Manola, Paul Ayris, Cameron Neylon, Pilar Rico, Michelle Barker, Ginny Barbour y un largo etcétera.

\section{REFERENCIAS}

Albers, Bianca; Shlonsky, Aron y Mildon, Robyn (2020). Implementation Science 3.0. https://doi.org/10.1007/978-3030-03874-8.

ALLEA (2017). The European Code of Conduct for Research Integrity. Revised Ed. Berlin: ALLEA - All European Academies. https://allea.org/wp-content/ uploads/2017/05/ALLEA-European-Code-of-Conduct-for-Research-Integrity-2017.pdf.

Allen, Christopher y Mehler, David M. A. (2019). Open Science Challenges, Benefits and Tips in Early Career and Beyond. PLOS Biology 17 (5): e3000246. https://doi.org/10.1371/journal. pbio.3000246.

Arrizabalaga, Olatz et al. (2020). Open Access of COVID-19-Related Publications in the First Quarter of 2020: A Preliminary Study Based in PubMed. F1000Research 9 (agosto): 649. https://doi. org/10.12688/f1000research.24136.2.

Ayris, Paul et al. (2018). Open Science and its role in universities: A roadmap for cultural change. LERU. Advice Paper 24. https://www.leru.org/files/LERUAP24-Open-Science-full-paper.pdf.

Barbour, Virginia y Borchert, Martin (2020). Open science: after the COVID-19 pandemic there can be no return to closed working. Policy \& Features. Australian Academy of Sciences. https://www. science.org.au/curious/policy-features/ open-science-after-covid-19-pandemicthere-can-be-no-return-closed-working.

Beaudry, Jennifer, Jordy Kaufman, Tom Johnstone, y Lisa Given. 2019. Swinburne
Open Science Survey (2019). https:// doi.org/10.17605/OSF.IO/VPWF7.

Belli, Simone et al. (2020). Coronavirus Mapping in Scientific Publications: When Science Advances Rapidly and Collectively, Is Access to This Knowledge Open to Society? Scientometrics, 124 (3): 2661-85. https://doi.org/10.1007/ s11192-020-03590-7.

Besançon, Lonni et al. (2020). Open Science Saves Lives: Lessons from the COVID-19 Pandemic. bioRxiv. The Preprint Server for Biology. https://doi. org/10.1101/2020.08.13.249847.

Bezjak, Sonja et al. (2018). Open Science Training Handbook. Zenodo. https:// doi.org/10.5281/ZENODO.1212496.

Bücheler, Thierry y Sieg, Jan Henrik (2011). Understanding science 2.0: Crowdsourcing and Open Innovation in the scientific method. Procedia Computer Science, 7: 327-329. https://doi.org/10.1016/j.procs.2011.09.014.

Burgelman, Jean-Claude; Osimo, David y Bogdanowicz, Marc (2010). Science 2.0 (change will happen....). First Monday, 15 (7). https://doi.org/10.5210/fm. v15i7.2961.

Burgelman, Jean-Claude et al. (2019). Open Science, Open Data, and Open Scholarship: European Policies to Make Science Fit for the Twenty-First Century. Frontiers in Big Data 2 (diciembre): 43. https://doi.org/10.3389/fdata.2019.00043.

Capps, Benjamin (2020). Where Does Open Science Lead Us During a Pan- demic? A Public Good Argument to Prioritize Rights in the Open Commons. Cambridge Quarterly of Healthcare Ethics, 30 (1): 11-24. doi:10.1017/ S0963180120000456

Chan, Leslie (ed.) (2019). Contextualizing openness: situating open science. Perspectives on open access. Ottawa: University of Ottawa Press.

Chretien, Jean-Paul; Rivers, Caitlin M. y Johansson, Michael A. (2016). Make Data Sharing Routine to Prepare for Public Health Emergencies. PLOS Medicine 13 (8): e1002109. https://doi. org/10.1371/journal.pmed.1002109.

Committee on Toward an Open Science Enterprise, Board on Research Data and Information, Policy and Global Affairs, y National Academies of Sciences, Engineering, and Medicine (2018). Open Science by Design: Realizing a Vision for 21st Century Research. Washington, D.C.: National Academies Press. https://doi.org/10.17226/25116.

Council of the European Union (2015). Council conclusions on open, data-intensive and networked research as a driver for faster and wider innovation. 9360/15 RECH 183 TELECOM 134 COMPET 288 IND 92. http://data.consilium. europa.eu/doc/document/ST-93602015-INIT/en/pdf.

David, Paul A. (2008). The Historical Origins of "Open Science": An Essay on Patronage, Reputation and Common Agency Contracting in the Scientific Revolution. Capitalism and Society 3 (2). doi:10.2202/1932-0213.1040 
David, Paul A. (2014). The Republic of Open Science: The institution's historical origins and prospects for continued vitality. EconPapers. http://econpapers.repec.org/paper/sipdpaper/13-037.htm.

David, Paul A.; Den Besten, Matthijs y Schroeder, Ralph (2006). How Open is e-Science? En 2006 Second IEEE International Conference on e-Science and Grid Computing (e-Science'06), 33-33. Amsterdam, The Netherlands: IEEE. https://doi.org/10.1109/E-SCIENCE.2006.261117.

Delaney, Niamh et al. (2020). European Commission, y Directorate-General for Research and Innovation. 2020. Science with and for Society in Horizon 2020: Achievements and Recommendations for Horizon Europe. Brussels: European Commission, Directorate-General for Research and Innovation. https:// op.europa.eu/publication/manifestation_identifier/PUB_KI0120165ENN.

ECSA (2015). Ten principles of Citzen Science. UCL Press. https://doi. org/10.14324/111.9781787352339.

European Commission (2015a). Validation of the results of the public consultation on Science 2.0: Science in Transition. Febrero de 2015. http://ec.europa.eu/ research/consultations/science-2.0/ science_2_0_final_report.pdf.

European Commission (2015b). A Digital Single Market Strategy for Europe. Communication from the Commission to the European Parliament, The Council, The European Economic and Social Committee and the Committee of Regions. COM (2015) 192 final. https:// eur-lex.europa.eu/legal-content/EN/ TXT/?uri=celex:52015DC0192.

European Commission (2016). Open Innovation, Open Science, Open to the World: A Vision for Europe. Luxembourg: Publications Office of the European Union.

European Commission Expert Group on FAIR Data (2018). Turning FAIR into Reality: Final Report and Action Plan from the European Commission Expert Group on FAIR Data. Brussels: European Commission. Directorate-General for Research and Innovation. https:// doi.org/10.2777/1524.

Fecher, Benedikt y Friesike, Sascha (2014). Open Science: One Term, Five Schools of Thought. En Opening Science: The Evolving Guide on How the Internet Is Changing Research, Collaboration and Scholarly Publishing, Sönke Bartling y Sascha Friesike, (ed.). Cham: Springer International Publishing, pp.17-47. https://doi.org/10.1007/978-3-31900026-8_2.

Gezelter, Dan (2011). An Informal Definition of OpenScience. The Openscience Project (blog). 2011. http:// openscience.org/an-informal-definition-of-openscience.

Gobierno de España. Ministerio de Ciencia e Innovación. 2020. EECTI: Estrategia Española de Ciencia, Tecnología e Innovación 2021-2027. Madrid: Ministerio de Ciencia e Innovación. https://www. ciencia.gob.es/stfls/MICINN/Ministerio/FICHEROS/EECTI-2021-2027.pdf.

Guédon, Jean Claude et al. (2019). Future of Scholarly Publishing and Scholarly Communication: Report of the Expert Group to the European Commission. Brussels: European Commission. Directorate-General for Research and Innovation. https://data.europa.eu/ doi/10.2777/836532.

Heise, Christian, y Pearce, Joshua M. (2020). From Open Access to Open Science: The Path From Scientific Reality to Open Scientific Communication. SAGE Open 10 (2): 215824402091590. https://doi. org/10.1177/2158244020915900.

Hocquet, Alexandre (2020). Open Science in Times of Coronavirus: Introducing the Concept of "Real-Time" Publication. Substantia, 4(1), 937 https://doi. org/10.13128/Substantia-937

Jhangiani, Rajiv S., y Biswas-Diener, Robert (ed.) (2017). Open: The Philosophy and Practices that are Revolutionizing Education and Science. London: Ubiquity Press. https://doi.org/10.5334/bbc.

Kuhn, Thomas S. (2012). The structure of scientific revolutions. Fourth edition, 50th anniversary edition. Chicago, London: The University of Chicago Press.

Kwon, Diana (2020). How Swamped Preprint Servers Are Blocking Bad Coronavirus Research. Nature 581 (7807): 130-31. https://doi.org/10.1038/ d41586-020-01394-6.

Lamprecht, Anna-Lena et al. (2020). Towards FAIR principles for research software. Paul Groth y Michel Dumontier (ed.). Data Science 3 (1): 37-59. https://doi.org/10.3233/DS-190026.

Laursen, Keld y Salter, Ammon J. (2014). The paradox of openness: Appropriability, external search and collaboration. Research Policy 43 (5): 867-78. https:// doi.org/10/gdj5w4.

Leonelli, Sabina; Spichtinger, Daniel y Prainsack; Barbara (2015). Sticks and carrots: encouraging open science at its source. Geo: Geography and Environment, 2(1): 12-16. https://dx.doi. org/10.1002\%2Fgeo2.2

Masuzzo, Paola y Martens, Lennart (2017). Do You Speak Open Science? Resources and Tips to Learn the Language. PeerJ Preprints 5:e2689v1 https://doi. org/10.7287/peerj.preprints.2689v1

Mayer, Katja (2015). From Science 2.0 to Open Science - Turning rhetoric into action? STCSN e-letter 3. https://sites. google.com/a/ieee.net/stc-social-networking/e-letter/stcsn-e-letter-vol-3no-1/from-science-2-0-to-open-science.

Méndez, Eva (2003). La descripción de documentos electrónicos a través de metadatos: una visión para la Archivística desde la nueva eAdministración. Revista d'Arxius, vol. 2003: 47-82. http:// arxiversvalencians.org/wp-content/ uploads/2020/04/revista2003_mendez.pdf; https://e-archivo.uc3m.es/ handle/10016/878.

Méndez, Eva, et al. (2020). Towards a shared research knowledge system: final report of the open science policy platform. Brussels: European Commission. Directorate-General for Research and Innovation. https://doi. org/10.2777/00139.

Merton, Robert K. (1974). The Sociology of Science: Theoretical and Empirical Investigations. Chicago: University of Chicago Press.

Miedema, Frank (2012). Science 3.0: real science, real knowledge. Amsterdam: Amsterdam University Press.

Ministère de L'Enseignement Supérieur de la Recherche et de L'Innovation (2020). A global strategy for open science: France's proposal on Open Science put forward in the framework of the Unesco consultation. https:// 
www.ouvrirlascience.fr/wp-content/ uploads/2020/08/Recommendation-UNESCO-Open-Science-France-Proposal.pdf.

Mons, Barend et al. (2016). Realising the European Open Science Cloud: First Report and Recommendations of the Commission High Level Expert Group on the European Open Science Cloud. Brussels: European Commission. Directorate-General for Research and Innovation. http://bookshop. europa.eu/uri?target=EUB:NOTICE:KI0116872:EN:HTML.

Montgomery, Lucy et al. (2018). Open Knowledge Institutions. MIT Press OA Books, julio. https://doi.org/10.21428/ 99f89a34.

Muscella, Silvana et al. (2018). Prompting an EOSC in Practice: Final Report and Recommendations of the Commission 2nd High Level Expert Group on the European Open Science Cloud (EOSC), 2018. LU: Publications Office. https:// data.europa.eu/doi/10.2777/112658.

National Research Council (2012). The Future of Scientific Knowledge Discovery in Open Networked Environments: Summary of a Workshop. Washington, D.C.: National Academies Press. https://doi.org/10.17226/18258.

Neylon, Cameron et al. (2019). Open Scholarship and the need for collective action. Zenodo. https://doi.org/10.5281/ ZENODO.3454688.

Nielsen, Michael (2011a). Reinventing Discovery: The New Era of Networked Science. Princeton: Princeton University Press.

Nielsen, Michael (2011b). Open Science Now! TedXWaterloo. https://www. ted.com/talks/michael_nielsen_open_ science_now.

O'Carroll, Connor et al. (2017a). Evaluation of Research Careers Fully Acknowledging Open Science Practices: Rewards, Incentives and/or Recognition for Researchers Practicing Open Science. Brussels: European Commission. Directorate-General for Research and Innovation. http://dx. publications.europa. eu/10.2777/75255.

O'Carroll, Connor et al. (2017b). Providing Researchers with the Skills and Competencies They Need to Practise Open
Science. Brussels: European Commission. Directorate-General for Research and Innovation. https://data.europa. eu/doi/10.2777/121253.

Ochoa-Gutiérrez, Jaider y Uribe-Tirado, Alejandro (2018). Ciencia abierta y bibliotecas académicas: Una revisión sistemática de la literatura. Open Science and Academic Library: A Systematic Literature Review. SSRN Electronic Journal. https://doi.org/10.2139/ ssrn.3483731.

OECD (2015). Making Open Science a Reality. OECD Science, Technology and Industry Policy Papers, vol. 25. https:// doi.org/10.1787/5jrs2f963zs1-en.

OSPP-REC (2018). Open Science Policy Platform Recommendations. Luxembourg: European Commission. Directorate-General for Research and Innovation.

Pardo Martínez, Clara y Poveda, Alexander (2018). Knowledge and Perceptions of Open Science among Researchers-A Case Study for Colombia. Information 9 (11): 292. https://doi.org/10.3390/ info9110292.

Pomerantz, Jeffrey y Peek, Robin (2016). Fifty shades of open. First Monday, 21(5). https://doi.org/10.5210/fm.v21i5.6360

Research and Information Network (2010). RINNews. RINews: Autumn 2010, Issue 11, http://www.rin.ac.uk/system/files/ attachments/RINews_Issue_11_0.pdf.

Roman, Mona; Liu, Jingwei y Nyberg, Timo (2018). Advancing the Open Science Movement through Sustainable Business Model Development. Industry and HigherEducation 32 (4): 226-34. https:// doi.org/10.1177/0950422218777913.

Saenen, Breg et al. (2019). Research Assessment in the Transition to Open Science: 2019 EUA Open Science and Access Survey Results. European University Association. https://eua.eu/ downloads/publications/research\%20 assessment\%20in\%20the\%20transition\%20to\%20open\%20science.pdf.

Scheliga, Kaja y Friesike, Sascha (2014). Putting open science into practice: A social dilemma? First Monday, 19(9). https://doi.org/10.5210/fm.v19i9.5381

Shaver, Lea Bishop (2009). The Right to Science and Culture. Wisconsin Law Review, 2010, 1: 121-84. https://doi. org/10.2139/ssrn.1354788.
Simeth, Markus, y Raffo, Julio D. 2013. What makes companies pursue an Open Science strategy? Research Policy 42 (9): 1531-43. https://doi.org/10/ f5gvwt.

Stahl, Bernd C. (2013). Responsible Research and Innovation: The Role of Privacy in an Emerging Framework. Science and Public Policy 40 (6): 708-16. https://doi.org/10.1093/scipol/sct067.

Stodden, Victoria (2010). Open science: policy implications for the evolving phenomenon of user-led scientific innovation. Journal of Science Communication 09 (01). https://doi. org/10.22323/2.09010205.

Teif, Vladimir B. (2014). On the Sociology of Science 2.0. En Opening Science: The Evolving Guide on How the Internet Is Changing Research, Collaboration and Scholarly Publishing, Sönke Bartling y Sascha Friesike (ed.), Cham: Springer International Publishing, pp. 309-311. https://doi.org/10.1007/978-3-31900026-8_23.

Tennant, Jonathan (2020). The [R]Evolution of Open Science. https://doi. org/10.5281/ZENODO.3700646.

Tennant, Jonathan et al. (2019). Foundations for Open Scholarship Strategy Development. Preprint MetaArXiv. https://doi.org/10.31222/osf.io/b4v8p.

Tyfield, David (2013). Transition to Science 2.0: "Remoralizing" the Economy of Science. Spontaneous Generations: A Journal for the History and Philosophy of Science 7 (1): 29-48. https://doi. org/10.4245/sponge.v7i1.19664.

United Nations (1948). Universal Declaration of Human Rights. https://www. un.org/en/universal-declaration-human-rights/.

Vicente-Saez, Rubén y Martinez-Fuentes, Clara (2018). Open Science Now: A Systematic Literature Review for an Integrated Definition. Journal of Business Research 88 (julio): 428-36. https://doi. org/10.1016/j.jbusres.2017.12.043.

Vignoli, Michela; Kraker, Peter y Sevault, Alexis (2015). Paving the Way for Science 2.0: Top-down and Bottom-up Approaches. Proceedings of the International Conference for E-Democracy and Open Government 2015. https:// doi.org/10.13140/RG.2.1.1943.0569. 
Von Schomberg, René (2012). Prospects for Technology Assessment in a framework of responsible research and innovation. En Technikfolgen abschätzen lehren: Bildungspotenziale transdisziplinärer Methode, Wiiesbaden: Springer VS. pp., 39-61. https://app. box.com/s/f9quor8jo1bi3ham8lfc.

Von Schomberg, René (2019). Why Responsible Innovation? En International Handbook on Responsible Innovation, René von Schomberg and Jonathan Hankins (ed.), Cheltenham: Edward Elgar Publishing, pp. 12-32. https://doi. org/10.4337/9781784718862.

Waldrop, Mitchell (2008). Science 2.0-Is Open Access Science the Future? Scientific American 298 (5): 68-73. https://doi.org/10.1038/scientificamerican0508-68.

Wehn, Uta et al .(2020). Global Citizen Science perspectives on Open Science: Written input by the CSGP Citizen
Science \& Open Science Community of Practice to the UNESCO Recommendation on Open Science. https://osf.io/ 6qjyg/?direct\%26mode=render\%26action=download $\% 26$ mode=render

Wilkinson, Mark D. et al. (2016). The FAIR Guiding Principles for scientific data management and stewardship. Scientific Data 3, 160018. https://doi. org/10.1038/sdata.2016.18

Willinsky, John (2005). The Unacknowledged Convergence of Open Source, Open Access, and Open Science. First Monday 10 (8). https://doi.org/10.5210/ fm.v10i8.1265.

Wilsdon, James et al. (2017). Next-Generation Metrics: Responsible Metrics and Evaluation for Open Science. Luxembourg: Publications Office. https:// data.europa.eu/doi/10.2777/337729.

World Health Organization (2015). Developing Global Norms for Sharing Data and Results During Public Health Emergencies. Statement arising from a WHO Consultation held on 1-2 September 2015. https://www.who.int/medicines/ebola-treatment/blueprint_phe_ data-share-results/en/.

Wouters, Paul et al. (2019). Indicator Frameworks for Fostering Open Knowledge Practices in Science and Scholarship. Brussels: European Commission. Directorate-General for Research \& Innovation. https://data.europa.eu/ doi/10.2777/445286.

Wyndham, Jessica M., y Weigers Vitullo, Margaret (2018). Define the Human Right to Science. Science 362 (6418): 975975. https://doi.org/10.1126/science. aaw1467.

Zastrow, Mark (2020). Open Science Takes on the Coronavirus Pandemic. Nature 581 (7806): 109-10. https://doi. org/10.1038/d41586-020-01246-3. 